\title{
Waves of desertification in a competitive ecosystem
}

\author{
Y. C. Daza C. ${ }^{* 1}$, M. F. Laguna ${ }^{\dagger 1}$, J. A. Monjeau ${ }^{\ddagger 3,4}$, and G. \\ Abramson $\S 1,2$ \\ ${ }^{1}$ Centro Atómico Bariloche and CONICET, R8402AGP Bariloche, Argentina \\ ${ }^{2}$ Instituto Balseiro, R8402AGP Bariloche, Argentina \\ ${ }^{3}$ Fundación Bariloche and CONICET, R8402AGP Bariloche, Argentina \\ ${ }^{4}$ Laboratório de Ecologia e Conservação de Populações, Departamento de Ecologia, \\ Universidade Federal do Rio de Janeiro, Rio de Janeiro, Brasil
}

February 1, 2019

\begin{abstract}
We study a mathematical model inspired by a common scenario in northern Patagonia consisting of humans and their livestock living together with native wildlife. The main production system in this area is sheep farming, which due to wrong historical management has led to desertification of the habitat, with impact on both native wildlife and livestock. Particularly the largest native herbivore, the guanaco, has reflected in their numbers this alteration induced in the environment. We analyze a mathematical model that captures the main characteristics of the interaction between sheep and guanaco: the hierarchical competition and the advantage granted by humans to the herds, and also incorporates a dynamic for the habitat. Using the metapopulation formalism, the trophic web of two herbivores is extended over a patchy landscape that considers two characteristic times for the dynamics of the resource. Our study stresses the dependence of the metapopulations dynamics on the recovery time of the resource. These results are backed up by a deterministic mean field model which shares some similarities with the stochastic and spatially extended one. We find different regimes depending on the parameters considered: coexistence of both species, survival of a single species and extinction of the other, and extinction of both. Remarkably, in some regions of parameters space we detect the presence of periodical spatio-temporal patterns, with persistent oscillations of constant amplitude. Based on these results, we perform a characterization of the observed scenarios in order to gain insight about the system.
\end{abstract}

\footnotetext{
*yudy.daza@cab.cnea.gov.ar

†lagunaf@cab.cnea.gov.ar

‡amonjeau@fundacionbariloche.org.ar

§abramson@cab.cnea.gov.ar
} 
Keywords - Livestock-wildlife coexistence, Hierarchical competition, Habitat destruction, Desertification, Metapopulations, Mathematical Modelling

February 1, 2019

\section{Introduction}

This model is based on a true story.

Indeed, the mathematical model we have developed is inspired by the history of the environmental devastation of Patagonia. Located in the southernmost tip of South America, in Argentina, the Patagonian steppe is an emblematic landscape 22. The extra-Andean Patagonia comprises c. $750000 \mathrm{~km}^{2}$ of arid and semiarid lands where extensive sheep ranching is the predominant land use [35, 26]. The unsustainable management of sheep farming is as old as the expulsion of native peoples by the Argentine army, just over 100 years ago, replacing a hunter-gatherer system of thousands of years by a system of private lands, subdividing a landscape, previously continuous, in ranches in turn divided internally by wire fences [6].

The basis of the subsistence economy of the original peoples of Patagonia was the use of native herbivores, especially the guanaco (Lama guanicoe) 24]. The guanaco has coevolved in the arid ecosystems, minimizing the environmental damage in the construction of its niche 31. As groups of guanacos constantly move in search of good pastures, there is a dynamic in which palatable and less palatable species alternate their abundance over time and space, allowing a sustainable foraging of the resource (when it occurs in natural conditions), and the concomitant control by predators, such as the puma (Puma concolor). The Tehuelche people built their niche learning to follow immense groups of guanacos, of up to 300000 individuals, in their seasonal migrations, a nomadic system that subsisted in a sustainable way for at least 6000 years 23 .

After the so-called "conquest of the desert" at the end of the nineteen century [6], sheep and guanaco began to coexist as competitors for the forage resource [3]. In the early days of sheep farming, with large areas and low population density, a system similar to that of nomadism was common. Shepherds on horseback carried the flocks through vast extensions in search of good pastures [7]. The main movement, as in the case of guanacos and Tehuelches, occurred between winter and summer. In summer high places were used letting the low valleys rest, which were used in winter. This system still prevails in certain areas of Patagonia, such as Payunia in the provinces of Neuquén and Mendoza.

In another modality, the holistic management, the ranches are exploited in a way that maximizes the harness of the biomass by the flocks 32. The most common modality is, however, livestock management in paddocks [17, where the aim is to maximize short-term gain [28]. Obviously, as the wildlife is out of the market, it is perceived as an impediment to the maximization of profits, which is the source of conflicts between productive activities and the conservation of native species [3, 30. Land use in Patagonia often implies negative consequences for wildlife and these are particularly evident when the removal of certain species is supposed to increase landholders' income or reduce production costs [5]. This conflict is exacerbated in arid rangelands or in periods of drought, where forage and water availability show high variability in space and time [38, intensifying wildlife prosecution [26]. Previous studies have addressed that due to direct and indirect competition, wild herbivores abundance is inversely correlated with livestock density [3, 29]. In particular, the 
interspecific competition of the guanacos with livestock lies in the fact that both herbivores are generalists and their diets overlap up to $83 \%$ 31, and landowners perceive a decrease in carrying capacity when guanaco abundance increases [25].

Given that the inadequate management of livestock is the general rule (having more animal load than the carrying capacity) the deterioration of the habitat, the loss of the species of greater nutritional value and the extinction of wildlife are the most common consequences of the current situation [12, 10].

Depending on the use of the land, the balance between wildlife and livestock use can be very different. In the few protected areas of Patagonia, which occupy only $4.62 \%$ of its surface [26] a focus on conservation is expected. In contrast, landholders relying on entirely productive activities often have no reason to offset the costs derived from wildlife tolerant practices 37. Therefore, when resources are limited, or international prices put pressure on the landholders, the competition of guanaco with sheep becomes very significant. In such circumstances it is expected that the main motivation of the ranchers is to solve the problem by exterminating or driving away wildlife, since it does not bring them any benefit 25 . It is necessary to understand how these anthropic factors affect decision making regarding the management of sheep farming and its coexistence with wildlife. In most ranches, top predators ( $P$. concolor and Lycalopex culpaeus) and herbivores (L. guanicoe, Pterocnemia pennata) have been locally extirpated by hunting or scare to reduce economic losses [26, 25] and to feed domestic dogs and cats [27, 11, 20, 36].

Despite the fact that the guanaco is highly fit to inhabit the Patagonian desert, its population density has diminished dramatically in historical times by having to share its natural environment with humans. If there were no human intervention, the guanaco herds, due to their size and natural history, would competitively impose on herds of sheep in the territorial struggle for resources such as food or water. But the current scenario tells an unnatural story. The flocks along with their guards (human, guns, dogs, fences: the livestock system) make difficult the survival of guanaco populations when the better pastures and wetlands are monopolized by human activities, and the populations are killed or displaced to marginal places with poor plant cover and nutrient deficiency; the same goes for other wildlife species, such as native deer [8, 9]. Then, the places occupied by the livestock suffers degradation due to the intrinsic foraging characteristics of the sheep and the wrong management plans that privileges short-term gains rather than long-term sustainability. As a result, many ranches were desertified and abandoned, ruining the natural capital for humans 2 and wildlife [4.

This introductory context is the basis for the assumptions we make in the dynamical model presented here. In a previous article we explored other aspects of the same problem with a similar model [18. In this work we analyze an ecological system inspired in the hierarchical competitive interaction of guanaco and sheep. The problem is addressed in the framework of metapopulations, which provides the possibility of a mechanistic understanding of the biological consequences of habitat loss and fragmentation [15, 16]. The occupation of patches is driven by ecological processes that occur in a stochastic way (extinction and colonization) and also are modulated by the patch state, which in turn is determined by the dynamics of the competitors. We show that an interesting dynamics arises, showing scenarios of coexistence, extinction and metapopulation waves. 


\section{Mathematical model}

We consider two species in hierarchical competition that affects an extended resource. As we said, the guanaco is competitively superior to the sheep due to its character and size, but human intervention makes the sheep better moving around and colonizing new patches. These features are included in the model through the rules of interaction and the value of the parameters chosen for each species.

Besides, the result of grazing is different for both species. The guanaco has coevolved with the flora they consume and, under natural conditions, there is a spatiotemporal dynamics of abundance among species of different palatability, allowing a sustainable consumption over time. For this reason, in the model, the impact of the guanaco on the habitat will be considered as negligible. The case of sheep is very different, since overgrazing can cause a strong impact on the resource, affecting both herbivores in a negative feedback. In such a context, the key factor that determines the survival of any species is the dynamics of the spatio-temporal structures that emerge from the ecological interactions.

\section{The metapopulation model for the two herbivores}

The classical approach of metapopulations consists of a system formed of local populations of a given species, located in specific places called patches [19, 14]. It is assumed that, for the survival of a local population, the patch must provide sufficient conditions to maintain and sustain them. Then, the patches are modeled like places suitable or unsuitable for be the habitat of a given species. The metapopulations are driven by ecological processes like death, colonization and interspecific competition. Competition can be captured by the model making the superior species (guanaco) and the inferior one (sheep) unable to share the same patch, as the inferior herbivore will be repealed from any patch already occupied by the superior one, or could be expelled if a superior migrates to the patch that is occupying. Colonization, on the other hand, will be determined by the human intervention. Although the guanaco is capable for surviving in regions where the sheep can not, the presence of the human makes the balance inclined towards the sheeps, as herds are protected and moved from one place to another. In this work we consider the colonizing capability of sheeps as a parameter always greater than the colonizing capability of the guanacos. Finally, deaths have to be understood as extirpation from a patch. This process can occur by a diversity of mechanisms, like death of the local population that occupy the patch, or migration to another patch, but the focus is always on the property of occupation of a patch. The extinction of a species in this context corresponds to their disappearance from all the patches.

We model the ecological processes that drive the dynamics just described as stochastic processes, in order to reflect the demographic fluctuations [15]. The dynamical variables are $x_{1}$ and $x_{2}$, the fraction of patches occupied by the superior and the inferior competitor, respectively. Local extinctions occurs at random with probabilities $e_{1}$ and $e_{2}$. Colonization takes place in the model according to the availability of patches in the immediate neighborhood of an occupied one. It is also a stochastic process characterized by $c_{1}$ and $c_{2}$. The availability of patches reflects both the dynamics of the resource (to be specified below) and the occupation by other populations. Given the hierarchical nature of the competitive interaction we consider two cases: 1) a patch can be occupied by the superior herbivore if it is free of their own, regardless of the presence of the inferior competitor; 2) a patch can be occupied by the inferior herbivore 


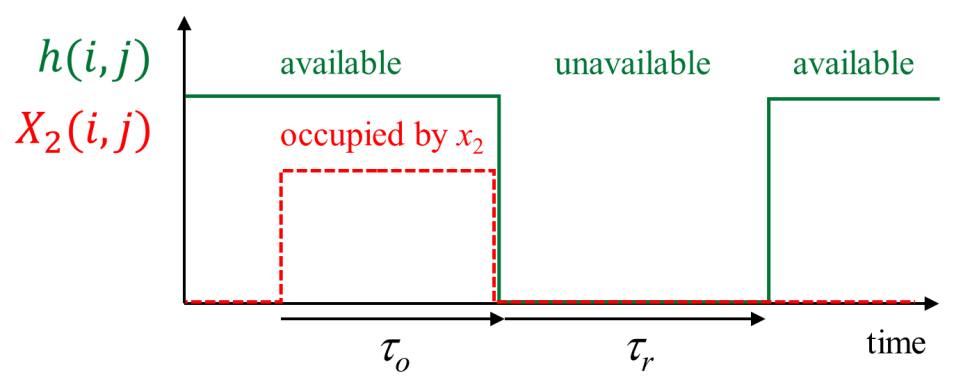

Figure 1: Dynamics of patch $(i, j)$. There are two characteristics times: the occupation time by the species 2 until the patch becomes unavailable $\left(\tau_{o}\right)$, and the recovery time $\tau_{r}$.

if it is free of both species. Further, in a patch occupied by both species, the inferior competitor can be displaced by the superior one with probability $c_{1}$. Together, these rules characterize the asymmetric (hierarchical) competition. A detailed discussion can be found in our previous works [18, 1.

\section{Dynamics of the habitat}

As stated above, a patch can be in one of two states: empty or occupied. This binary state would fluctuate at each patch according to the dynamics of the metapopulations. Besides, the resources at each patch can change according to a number of processes, such as the renewal of the pastures according to environmental factors like temperature, precipitation, etc., as well as the consumption by both species. Depending of the pressure of grazing the resource, $h$, may remain sustainable or become depleted. If the continuous occupation by the inferior competitor depletes $h$ at a patch, that patch becomes unsuitable for colonization for both $x_{1}$ and $x_{2}$. In an optimistic scenario, we can assume that the resource has the power to recover the pre-occupation level.

We have implemented the dynamics described above in a simplified way, with a single resource $h$. We use two time scales, characteristic of the depletion by overgrazing $\left(\tau_{o}\right)$ and the recovery of the resource $\left(\tau_{r}\right)$. An internal clock at each patch takes care of the state of $h$. It starts counting at the colonization by the inferior competitor, and after a lapse $\tau_{o}$ of continuous occupation the resource is set to zero, local populations get extinct and the patch is flagged as unavailable for colonization ("desertification"). After a time $\tau_{r}$ the resource is recovered, and the patch is again suitable for colonization. Figure 1 shows a schematic representation of this dynamics.

\section{Numerical implementation}

In order to understand the effects of the state of the habitat on the occupation by the species, a spatially extended metapopulation model was implemented. The trophic web is embedded in a space structured like a square grid of side $L$. The borders of the grid are set as unsuitable for occupation, meaning that border conditions are closed. The possible states of each patch are: suitable or unsuitable for occupation ( $h=1$ or 0 respectively). The patches are initially all set at the $h=1$ state and both species occupy them in the same proportion and randomly distributed. If a population 
inhabits a patch, the variable of occupation $X_{k}(i, j)$ takes the value 1 (here $k \in\{1,2\}$ is an index for the species, and $0<i, j<L$ are indices of position of the patch in the grid). If the species does not occupy the patch, $X_{k}(i, j)=0$. Because of the dependence of the state of the patches on previous occupation, a history is necessary at the start of the simulation. We also generate this at random, since the dynamics eventually overcomes any transient state. Time runs in a discrete way and at each time step the ecological processes occur in a stochastic way.

We run numerical simulations during a number of iterations that guarantees that the system reaches a stationary state in which the fraction of patches occupied by species $k$, defined as $x_{k}=\frac{1}{L^{2}} \sum_{i, j} X_{k}(i, j)$, fluctuates around a well defined value. In all the simulations we use $L=100$.

\section{Results}

In this section we present the main results obtained with the numerical implementation of the stochastic and spatially extended version of the model. We will leave for the next section the description of the results of the mean field model.

\section{Evolution of the metapopulations}

Figure 2 shows the behavior of typical populations of the two herbivores for several values of the occupation time $\tau_{o}$. The remaining parameters, $c_{1}, c_{2}, e_{1}, e_{2}$ and $\tau_{r}$, were held fixed at the values indicated in the caption of the figure. In the graphs we plot the fraction of patches occupied by both species $\left(x_{1}\right.$ for the superior herbivore and $x_{2}$ for the inferior one) at each time iteration. Each curve corresponds to a value of occupation time $\tau_{o}$. The color code goes from cold (small $\tau_{o}$ ) to warm colors (large $\left.\tau_{o}\right)$.

For small $\tau_{o}$ we observe a high level of occupation of the superior species $\left(x_{1} \approx 0.7\right.$ for $\tau_{o}=5$ ), which decreases abruptly as $\tau_{o}$ increases; see the left panel of Fig. 2 . In particular, for $11 \leq \tau_{o} \leq 40$ we obtain $x_{1}=0$, i.e. the superior species is extinct. When $\tau_{o}$ increases further, the fraction $x_{1}$ gradually grows, reaching values similar to those obtained for the smallest $\tau_{o}$ studied. In the right panel of Fig. 2 we present the behavior of the inferior species. The fraction $x_{2}$ grows with increasing $\tau_{o}$ as the curves, from bottom to top, go from cold to warm colors. Moreover, we observe an oscillatory behavior for a range of values of the occupation time $\tau_{o}$, meaning that the patches are occupied and vacated by the $x_{2}$ population with a constant global period.

Figure 2 helps to draw the big picture of how the occupations evolve, and allows to illustrate three possible regimes in the dynamics of a given species, namely:

1. extinction $\left(x_{k}=0\right.$, i.e. emptying of all the patches),

2. constant (non-oscillatory) survival $\left(x_{k}>0\right.$, fluctuating around a mean value but with no oscillations), and

3. oscillatory regime $\left(x_{k}>0\right.$, oscillating around a mean value with a well defined period).

An exhaustive description of the oscillatory regime demands a visualization of the spatial grid in which the species are immersed. We plot in Fig. 3 some snapshots of the patches during the evolution of the system. It is clear that, even though the system only has local interactions (extinction, colonization and competitive exclusion involve at most a local neighborhood of a patch), a global dynamics of the system is 

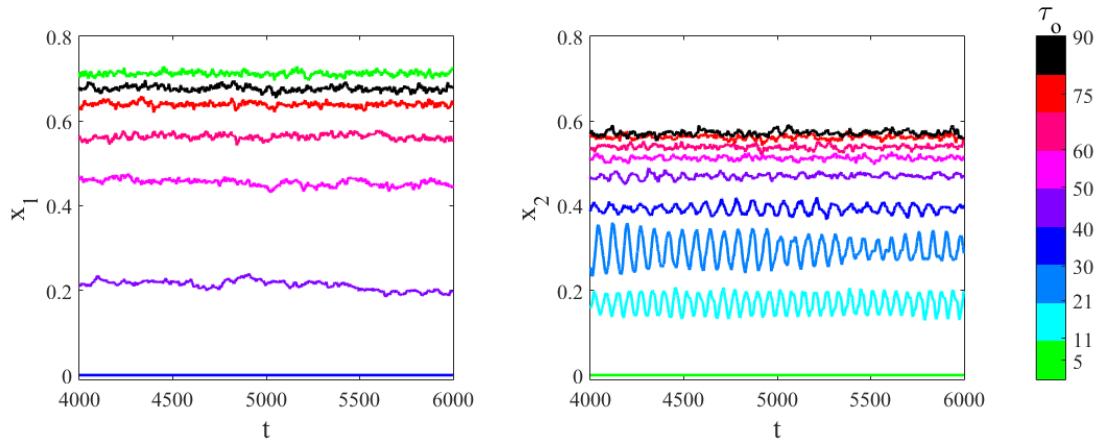

Figure 2: Temporal evolution of the fraction of patches occupied by the superior species $\left(x_{1}\right.$, left panel) and the inferior one $\left(x_{2}\right.$, right panel) for several values of occupation time $\tau_{o}$. Colors indicate the value of $\tau_{o}$, going from cold (for low values of $\tau_{o}$ ), to warm ones (for high values of $\tau_{o}$ ). Parameters used: $c_{1}=0.05$, $c_{2}=0.7, e_{1}=0.05, e_{2}=0.01, \tau_{r}=50$.

attained by the propagation of waves of colonization and desertification. To complete the picture of this regime, the central panel of Fig. 3 shows the fraction of patches occupied by the two herbivores together with the evolution of the resource. The existence of such periodical behavior suggests that we can characterize the different regimes by the frequency content of the occupations.

\section{Characterization of the regimes}

In order to determine the frequency spectrum of the time series of the occupations we calculate their Fourier transforms. The principal frequency (the most intense frequency of the spectrum), if present, indicates an oscillatory behavior of the system, meaning that the occupation is changing with a well defined period. In Fig. 4 we plot the Fourier transforms of the curves of Fig. 2, calculated during the final 1500 time iterations. In correspondence with Fig. 2 the color scheme goes from cold colors (small $\tau_{o}$ ) to warm colors (large $\tau_{o}$ ). The spectra depict the presence of a periodicity in the occupations, even in the cases in which their evolution is rather noisy. In a wide region of the parameters space the spectra show the presence of a well defined frequency (and their harmonics) mounted on a basal signal originated in the stochastic noise intrinsic to the biological processes.

Although the detection of a principal peak in the Fourier transform indicates an oscillatory behavior, its presence is dubious in some cases because its intensity is very small and can not be separated from noise in a efficient way. For this reason we set up a phenomenological procedure that can be easily programmed to detect peaks automatically. From each spectrum we removed an exponentially fitted background, which leaves mainly the peaks if present. After this we run an automatic detection of peaks, and the spectrum was labelled as oscillating if it has a peak above a threshold. We set this threshold as $1 \%$ of the amplitude of the largest peak for the corresponding parameters. This small value prevents overlooking the oscillations of $x_{1}$ even in the region where $x_{2}$ is synchronized with the resource (as in Fig. 4). It also allows a proper characterization of the decay of the amplitude of the oscillations as a function 

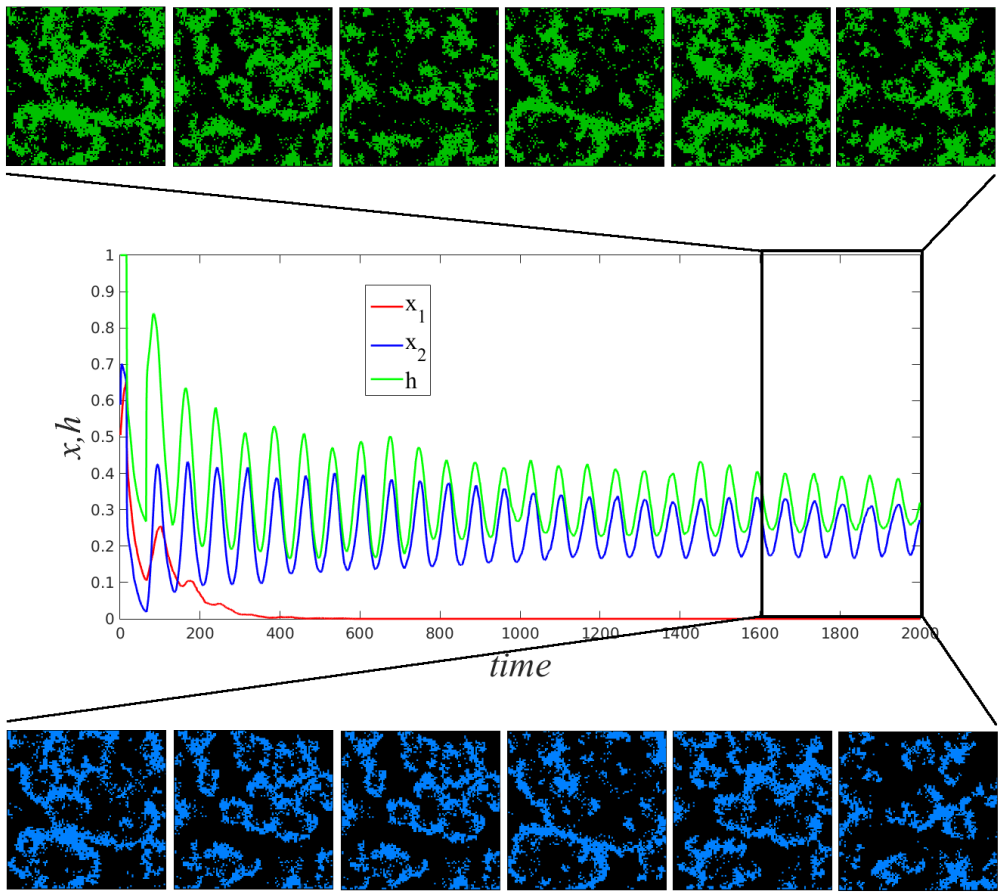

Figure 3: Top: Snapshots of the resource grid in the oscillatory regime. Available patches $(h=1)$ are painted green. Center: Temporal evolution of the species and the resource for the same parameters. The inferior species oscillates with the same period as the resource, whereas the superior one was extinguished in all the patches $\left(x_{1}=0\right)$. Bottom: Snapshots of the patches occupied by the inferior herbivore at the same time steps as the bottom panel. Patches occupied by $x_{2}$ are painted blue. Parameters used: $c_{1}=0.05, c_{2}=0.5, e_{1}=0.05$, $e_{2}=0.01, \tau_{r}=50$ and $\tau_{o}=17$.

of $\tau_{o}$, as shown below. With the information given by the first peak of the Fourier transform, we carried out a sweep in the phase space of the parameters of the model. The results obtained are presented if Fig. 5 in the following way: for a constant value of recuperation time $\tau_{r}$, we measure the frequency and height of the maximum of the first significant peak. These features are codified in the size and color of the circles, respectively. Data are plotted as a function of the occupation time $\tau_{o}$ for each colonization rate $c_{2}$.

Figure 5 shows that, in all cases, the increase in the occupation time $\tau_{o}$ produces a decrease in the size of the circles (which encodes the frequency value of the main peak). That is, the frequency of occupation waves decreases with the occupation time $\tau_{o}$. It is worth mentioning that the boundary between the zone of oscillations and the one of no oscillations is not abrupt but smooth, causing the border between these two regions to be blurred. For the purposes of presenting the data, such zones are 

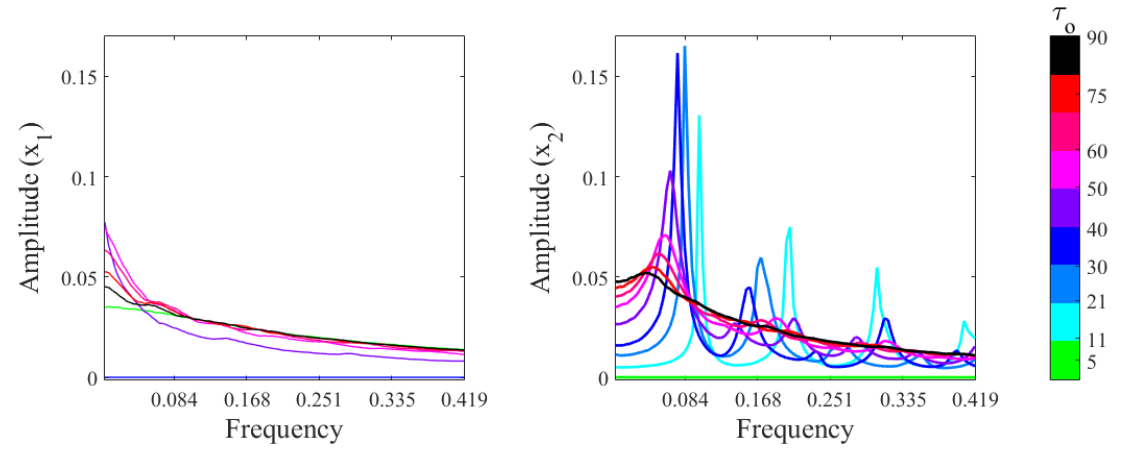

Figure 4: Left: Discrete Fourier transform of population $x_{1}$. Right: Discrete Fourier transform of population $x_{2}$. Parameters used: $c_{1}=0.05, c_{2}=0.7$, $e_{1}=0.05, e_{2}=0.01, \tau_{r}=50$. Colors go from cold, corresponding to low values of $\tau_{o}$, to warm colors, corresponding to high values of $\tau_{o}$.

drawn taking into account the threshold described above. It can also be observed that there are zones of extinction for either population, where the occupations of patches are null. The extinction regions move to large values of $\tau_{o}$ and they widen when the recuperation time of the patches $\tau_{r}$ increases.

Comparing the two top panels of Fig. 5 it can be observed that in the zones where the population of the superior herbivore is extinct from all patches $\left(x_{1}=0\right), x_{2}$ has oscillations with large amplitude (lighter color). Moving to larger $\tau_{o}$, the amplitude of $x_{1}$ increases while the amplitude of $x_{2}$ decreases. Both amplitudes gradually decrease to low values in regions of large $\tau_{o}$. Note also the difference in the scales of the amplitude of oscillations of $x_{1}$ and $x_{2}$. The presence of non-zero size circles and the scale of the intensity of the peaks in Fig. 5 illustrates that there are oscillations in the $x_{1}$ population for intermediate values of $\tau_{o}$, with a very small intensity when compared with those of $x_{2}$; however, they can not be ignored. The same can be said about the lower panels of Fig. 5, with the difference that the amplitudes change in a more homogeneous way in the zone post-extinction.

Figure 5 confirm the existence of three dynamical regimes for each population. There are extinction zones where the populations disappear from the system, oscillation zones where the populations evolve forming traveling waves over the patches, and regions where the occupation reaches a non-oscillatory steady state. We will return to this phase diagram in the Discussion, where we will carry out a more detailed analysis of the different scenarios.

\section{Mean field approximation}

It is possible to formulate a deterministic mean field model which shares some similarities with the stochastic and spatially extended one defined above. In the spirit of the original Levins model of metapopulations and its generalizations [19, 13] we propose the following delayed differential system, in terms of the density of patches $x_{1}(t)$ and $x_{2}(t)$, occupied by the superior and the inferior competitor respectively, and the available habitat $h(t)$ : 

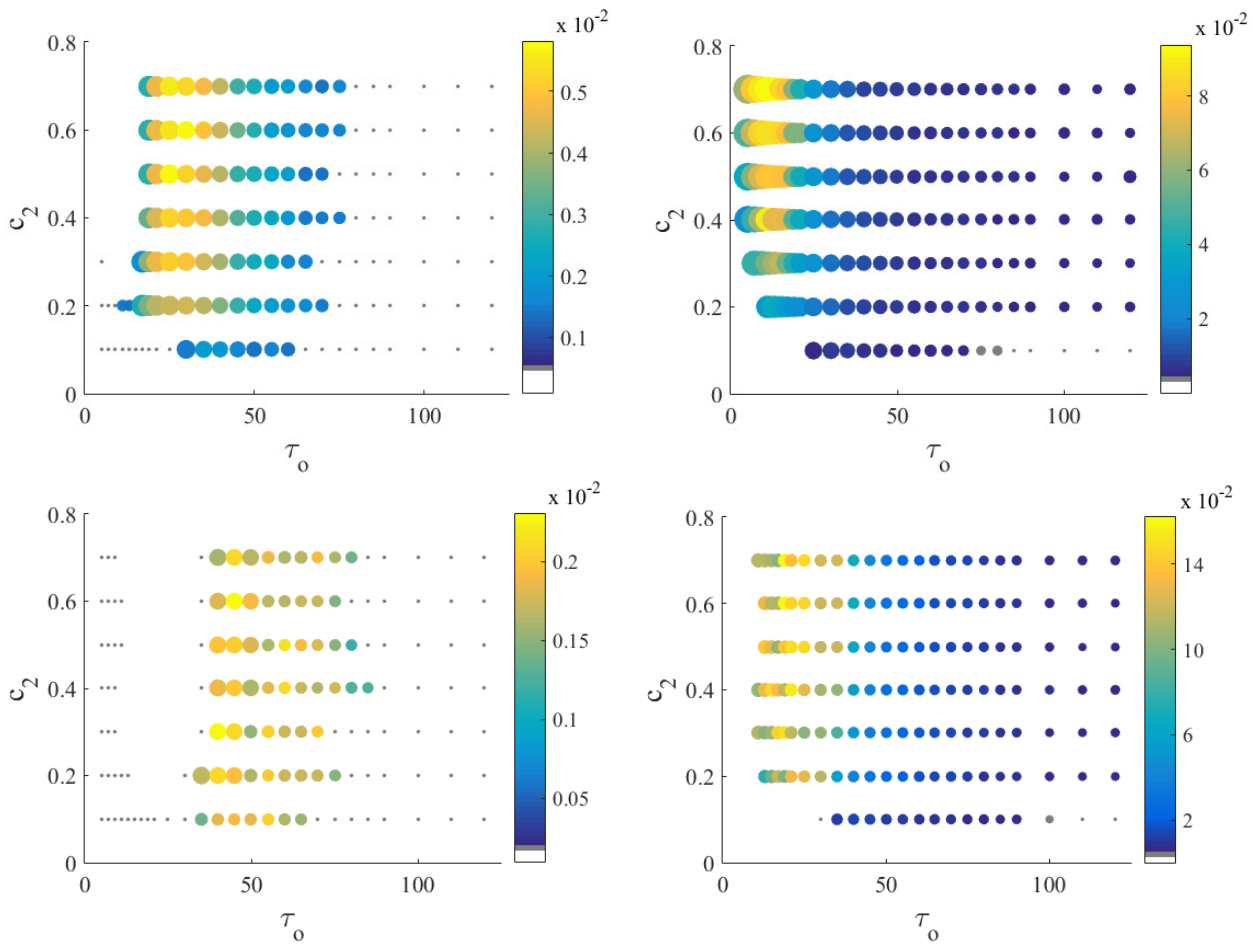

Figure 5: Phase space for the parameters colonization rate $c_{2}$ and occupation time $\tau_{o}$ for a constant value of the patch recuperation time $\tau_{r}$. Left: $x_{1}$. Right: $x_{2}$. Top: $\tau_{r}=10$. Bottom: $\tau_{r}=50$. The size of the circles codifies the frequency and their color codifies the height of the maximum of the first significant peak. The absence of circles corresponds to extinction and small gray dots indicate non-oscillatory steady state. Please note that the color scales of $x_{1}$ and $x_{2}$, showing the height of the peaks, are not the same: for a given color, the peak of $x_{1}$ is smaller.

$$
\begin{aligned}
\dot{x_{1}} & =c_{1}\left(h-x_{1}\right) x_{1}-e_{1} x_{1} \\
\dot{x_{2}} & =c_{2}\left(h-x_{1}-x_{2}\right) x_{2}-e_{2} x_{2} \\
\dot{h} & =-\gamma x_{2}\left(t-\tau_{o}\right) h\left(t-\tau_{o}\right)+\gamma x_{2}\left(t-\tau_{o}-\tau_{r}\right) h\left(t-\tau_{o}-\tau_{r}\right)
\end{aligned}
$$

In this system, the parameters $c_{1}, e_{1}$ and $c_{2}, e_{2}$ represent the colonization and (local) extinction rates of the respective species. Equation (3), representing the dynamics of the habitat, contains the delayed effect exerted by the species of the inferior competitor, responsible for the desertification. Observe that there is a negative contribution to the change of $h$, given by the occupied patches a time $\tau_{o}$ before $t$ (at a rate $\gamma$ ). There is also a positive one corresponding to the recovery of the destroyed habitat, after a time $\tau_{o}+\tau_{r}$ has elapsed. 

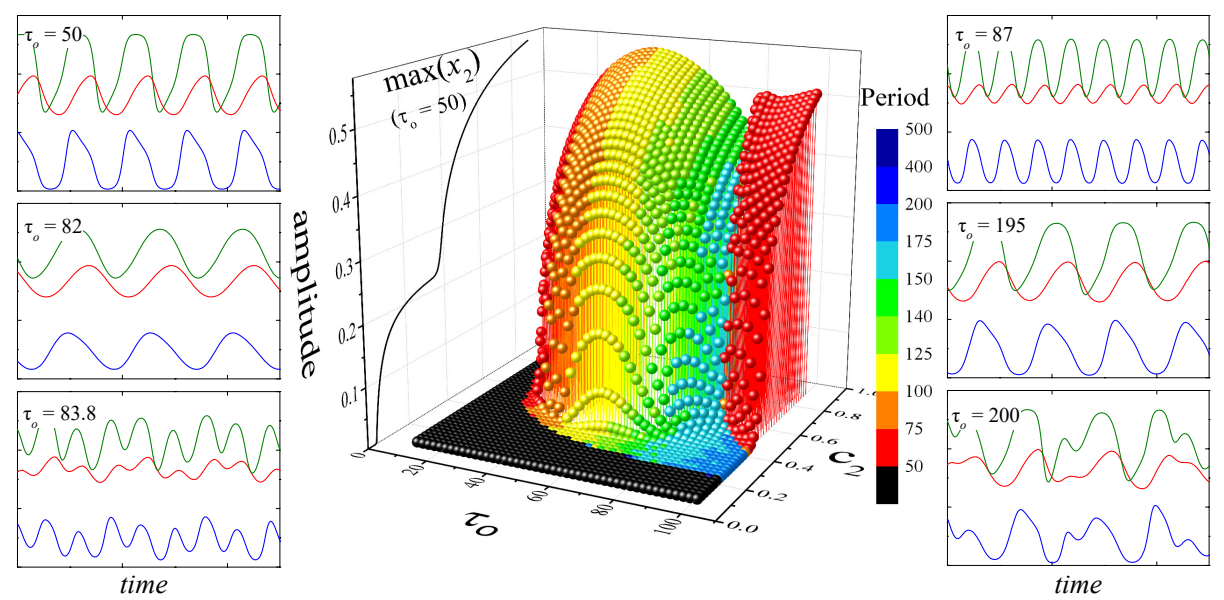

Figure 6: Phase diagram of the system (113). Fixed parameters are: $\tau_{r}=10$, $c_{1}=0.04, c_{2}=0.7, e_{1}=e_{2}=0.01, \gamma=0.1$. The lines show the evolution of $x_{1}$ (blue), $x_{2}$ (red) and $h$ (green).

It is important to stress that a model such as this ignores the short range correlations between occupied patches, which arise from the local and nearest neighbors dynamics of the extended system. This correlations play an important role in the most interesting behaviors of the spatial organization of the occupied space. Also, it ignores the role of fluctuations in the system. Nevertheless, some global features of the metapopulation dynamics can be captured by such an analytical model, and it is thus worth consideration.

We show in the central panel of Fig. 6 a representation of the typical dynamical phases present in the system. The horizontal plane spans the parameters $c_{2}$ and $\tau_{o}$, while the rest of the parameters remain fixed. The vertical coordinate represents the amplitude of the $x_{2}(t)$ solution of the system after a steady state is achieved. Fixed points have amplitude zero and are represented by the flat floor of the graph. A line of Hopf bifurcations separates these fixed points from (three-dimensional) cycles, of which the amplitude of the $x_{2}$ variable is shown as height. These cycles are also characterized by their period, shown as a color scale. The leftmost wall of the plot shows a cut of this landscape at the intermediate value $\tau_{o}=50$. This curve represents the maximum of $x_{2}$, and serves the purpose of showing the distinction between two phases separated by a transcritical bifurcation in the fixed points region: the extinction of $x_{2}$ (a narrow band close to $c_{2}=0$ ) and a coexistence of the two species with a positive $x_{2}$.

Besides these two bifurcations, there is a rich region of complex cycles attained by duplication of the period and chaos, separated from the simple limit cycles by a trough at $\tau \approx 80$ in Fig. 6. We have not explored this regime further, since in the present work our main interest is the behavior of the stochastic and spatially extended model, which more closely represents the situations found in real ecosystems. Some typical solutions are shown in the lateral panels. The period characterizing these phases (according to the colored scale) is the one with a strongest peak in Fourier space (which is just the period of the orbit in the case of simple cycles, before the period doubling), and the amplitude is the mean of the subcycles. 


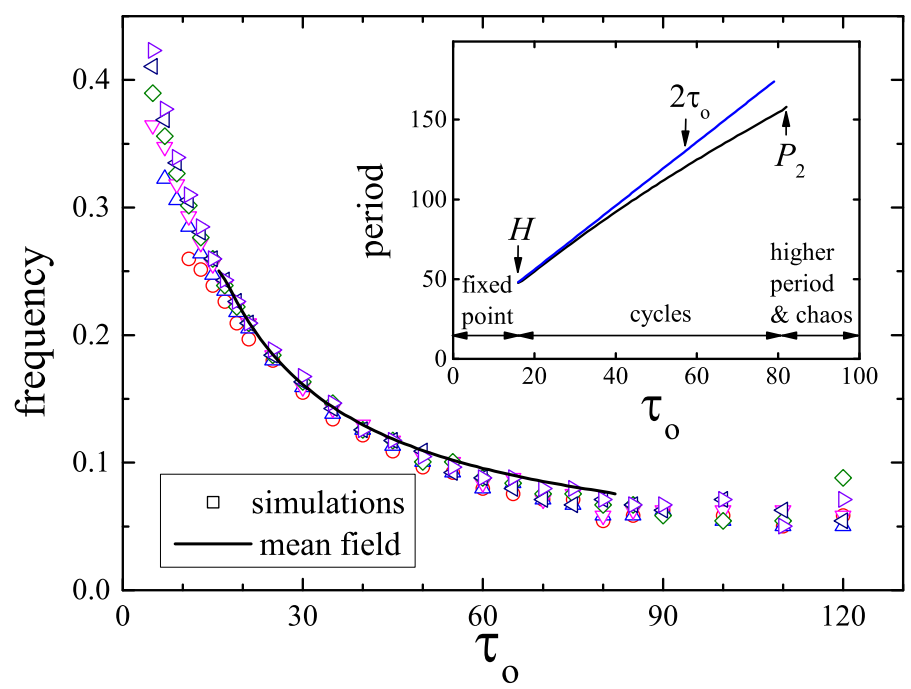

Figure 7: Diagram of the principal frequency of the occupation waves for $x_{2}$ in function of the occupation time $\tau_{o}$ for several values of colonization rate $c_{2}$. The inset illustrates the analogue situation in the mean field approximation. In this case, the $y$ axis indicates the period of the occupation waves.

As a final characterization of the oscillations displayed by the mean field model, we have plotted in the inset of Fig. 7 the period of the cycles as a function of the characteristic time $\tau_{o}$, corresponding to the same parameters as in Fig. 6 and $c_{2}$ fixed at 0.7 . The plot shows the range of simple limit cycles, from the point marked $H$ (the Hopf bifurcation) up to $P_{2}$ (the first period doubling bifurcation). After $P_{2}$ there exists the complex regime that we mentioned above, which is not displayed.

We can see that the period of the metapopulation solution grows with $\tau_{o}$ - as it should - in the region between the Hopf bifurcation and the period doubling. It is remarkable that this dependence is not linear (a straight line of slope 2 is shown for comparison). In other words, increasing the characteristic time of desertification does not produce a proportional increase in the period of the population oscillation. A phenomenological allometric function of the form:

$$
\text { period }=a+b \tau_{o}^{c}
$$

gives an excellent fit to the measured period with $c=0.74 \pm 0.04$, with an $R^{2}=0.99997$ confidence in the whole range of this phase. It is not easy to give a simple explanation for this departure from linearity. A sensible rationale behind it should consider that both phenomena, desertification and recovery, take place simultaneously in the system. While part of the system is becoming unavailable due to overgrazing, some other parts are already recovering from previous desertification events. The local population moves around the system (the metapopulation waves discussed above), allowing faster recovery of the occupation fractions, which is reflected in that the period bends down from a straight line as a function of $\tau_{o}$.

The main plot of Fig. 7 shows the corresponding frequencies of the stochastic simulation in the region of oscillations (reported in the previous section), also as function 
of $\tau_{o}$. Each set of points corresponds to a different value of $c_{2}$. We can see that the behavior is very similar to the one displayed by the mean field model (the black line). We have not observed the period doubling or the chaotic regimes in the simulations, most probably obscured by the fluctuations. Observe, nevertheless, an increase of the frequency (in some of the curves) for the largest values of $\tau_{o}$. Since these are the frequencies of the largest Fourier mode, this increase may be indicative of the regime of higher periods.

\section{Discussion}

In this work we have analyzed, in the framework of metapopulations, an ecological system inspired by a trophic web of two species of herbivores in hierarchical competition affecting an extended resource. For the populations of herbivores, three dynamical regimes were obtained in stochastic simulations as well as in a mean field approximation: extinction, oscillatory state, and non-oscillatory steady state.

The mean field model shows, for some values of the parameters, the existence of fixed points, corresponding to extinction or non-oscillatory behavior, and a the transition to a regime of cycles. These regimes are also observed in the stochastic model. The comparison between the two approaches can not be pushed too far, mainly because the mean field model does not take into account local interactions, spatial correlations and noise. Although it is expected that the long-range interactions inherent to the mean field contribute to synchronization, this is not the case for the stochastic (automata-like) model, with only local interactions. Therefore, it is remarkable that the synchronization of patches in the whole system is obtained in this case, by means of the drive imposed by the resource, which is in turn modulated by the behavior of the lower species $x_{2}$. The noise inherent to the probabilistic character of the biological processes also difficult the comparison of the results of both models. The chaotic behavior could be present in the automata model, but it is probably hidden by the noise, since it has been observed in other ecological models that were analyzed from the point of view of coupled maps lattices. In such systems the temporal evolution is obtained by means of maps that comprise terms of growing, intra- and interspecies competition, and coupling with local neighbors included as a diffusive operator [33, 34. In that case the system shows the existence of chaotic attractors; spatial structures are formed even with the combination of parameters that gives chaotic dynamics 33 .

In order to illustrate the observed dynamical behaviors, we plot in Fig. 8 the phase space of Fig. 5 but now the different regions have been colored. Dark regions are the extinction zones. The remaining ones are colored with tones that differ in intensity, being more saturated in the zone of oscillations and less saturated in zones where the mean occupations come to a steady value (with no oscillations).

By comparing the two top panels of Fig. 8, it can be seen that in the regions where $x_{2}=0$ (extinction of the inferior species), the population of the superior one $x_{1}$ comes to a non-oscillatory steady value. These results are obtained for low values of occupation time $\tau_{o}$, and low values of $c_{2}$, the capability of colonization of $x_{2}$, which make them unable to survive. Precisely, the absence of oscillations is due to the extinction of $x_{2}$, which in our model is the only one that affects the resource. That would be the state of the native species $x_{1}$ and the resource in the absence of the exotic species.

The same can be said when the bottom panels of Fig. 8 are compared. For both species the extinction zones enlarge when the recuperation time of the patch $\tau_{r}$ in- 

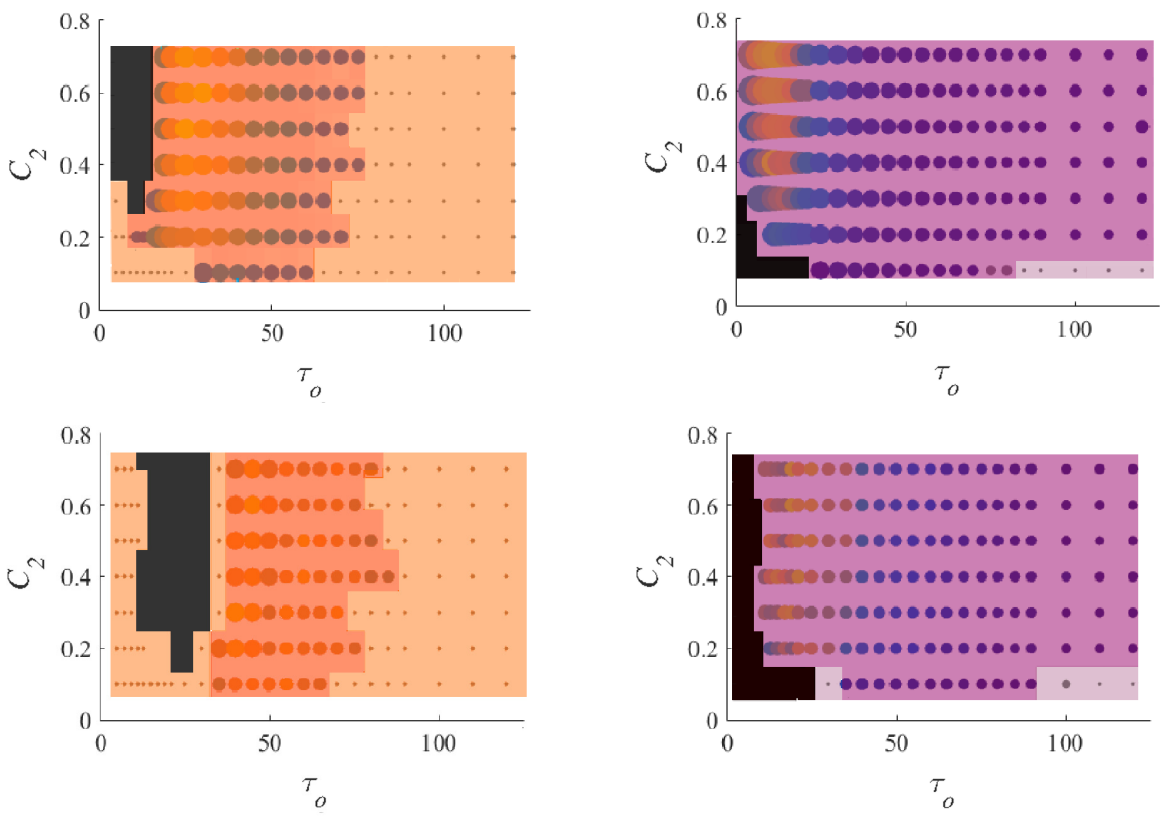

Figure 8: Phase space of the parameters $c_{2}$ and $\tau_{o}$ (colonization rate and occupation time) for a constant value of the patch recuperation time $\tau_{r}$. Left panels: $x_{1}$. Right panels: $x_{2}$. Top panels: $\tau_{r}=10$. Bottom panels: $\tau_{r}=$ 50. Colors represent the regimes present in the dynamics: saturated colors for oscillatory regions, softer colors for non-oscillatory steady state, and black for extinction.

creases. Specifically, in the case of $x_{2}$, when the patches take more time to recover, even if $x_{2}$ is a better colonizer (a larger $c_{2}$ ), it is not enough to avoid extinction.

It can also be observed that the phase space has zones where both populations oscillate together, which occurs because the presence of $x_{2}$-directly coupled with the resource-induces oscillations in the patches that in turn produce oscillations in $x_{1}$. In fact, we can observe more clearly in Fig. 5 that, for the parameters where $x_{1}$ is extinct, $x_{2}$ oscillates with a very large amplitude. In a real situation this could result into a very fluctuating income, which would not be a desired behavior for a profitable management.

There is also a region where the $x_{2}$ population does not exhibit oscillations for large values of the occupation time $\tau_{o}$. To understand what happens, observe the corresponding parameters there (lower right corner of the $x_{2}$ plots). The time $\tau_{o}$ can be seen alternatively as a measure of the environmental damage produced by the $x_{2}$ exotic population, because a longer $\tau_{o}$ means that $x_{2}$ is allowed a longer continuous presence at a patch (remember the patch comes to the $h=0$ state when the occupation time threshold $\tau_{o}$ is reached) and vice versa; then a longer $\tau_{o}$ results from a less harmful $x_{2}$. In other words, in this region $x_{2}$ cannot colonize so much (small $c_{2}$ ), but still survives because they are less harmful, plus they do not affect much the resource and then do not exhibit oscillations. Then, since the resource is not much affected by $x_{2}$, the native 
population $x_{1}$ can stay in a scenario near the natural conditions (without the exotic species), with long period oscillations of small amplitude. This is an unlikely scenario because it requires not so damaging herds.

In summary, if the occupation time $\tau_{o}$ is short, $x_{2}$ goes extinct if $c_{2}$ is small; but if $c_{2}$ increases, the population of $x_{1}$ becomes extinct. This situation gets worse if the recovery time, $\tau_{r}$, is longer. When $\tau_{o}$ is long the species coexist in a state where $x_{1}$ does not oscillate and $x_{2}$ show oscillations of low intensity and long period. For intermediate values of $\tau_{o}$, both species coexist exhibiting oscillations which increase their periods and decrease their intensities as either $\tau_{o}$ or $\tau_{r}$ increase.

\section{Concluding remarks}

The mathematical model presented here reproduces the scientific evidence on the causes of desertification, whose main driving force is not natural conditions but due, almost purely, to the sum of local and short-term decisions 2. This transforming force is characterized by the predominance of "I-here-now" over the ecologically correct pathway in the long term [21] and has, as a consequence, a negative feedback between the sheep farming and the pastures that sustain it, causing the loss of productivity, biodiversity, quality of life; and after the collapse, the abandonment of a useless land.

In this context, our mathematical model can help decision makers see "a movie" that shows the catastrophic future that awaits them, unless they change their way of thinking and management of land use.

\section{Acknowledgements}

We acknowledge financial support from several sources: Universidad Nacional de Cuyo (06/C506), ANPCyT (PICT-2014-1558) and CONICET (PIP 2015/0296).

\section{References}

[1] Abramson, G., Laguna, M. F., Kuperman, M. N., Monjeau, J. A., Lanata, J. L., 2017. On the roles of hunting and habitat size on the extinction of megafauna. Quaternary International 431, 205-215.

[2] Andrade, L., 2013. La mirada sociológica sobre la desertificación en la meseta central santacruceña (Patagonia austral, Argentina). Zonas Áridas 15(2), 402417.

[3] Baldi, R., Albon, S., Eston, D., 2001. Guanacos and sheep: evidence for continuing competition in arid patagonia. Oecologia 129, 561-570.

[4] Bonino, N., 2005. Guía de los mamíferos de la Patagonia Argentina. Ediciones INTA, Buenos Aires.

[5] Chaikina, N., Ruckstuhl, K., 2006. The effect of cattle grazing on native ungulates: the good, the bad, and the ugly. Rangelands 28(3), 8-14.

[6] Delrio, W., 2005. Memorias de expropiación. Sometimiento e incorporación indígena en la Patagonia 1872-1943. Universidad Nacional de Quilmes, Buenos Aires. 
[7] Fernandez, T., Gavirati, M., Jones, N., 2008. Eran todos campos abiertos. poblamiento y configuración del espacio socio-económico-cultural de península valdés (1880-1980). Cuad. Hist. Patagon. 2, 17-95.

[8] Flueck, W., Smith-Flueck, J., 2006. Predicaments of endangered huemul deer, Hippocamelus bisulcus, in Argentina: a review. Eur. J. Wildl. Res. 52(2), 69-80.

[9] Flueck, W., Smith-Flueck, J., Monjeau, J., 2011. Protected areas and extensive productions systems: a phosphorus challenge beyond human food. BioScience $61(8), 582$.

[10] Fuhlendorf, S., Engle, D., 2001. Restoring heterogeneity on rangelands: ecosystem management based on evolutionary grazing patterns. Bioscience 51(8), 625-632.

[11] Gallardo, G., Nuñez, A., Pacheco, L., Ruiz-García, M., 2009. Conservación del puma en el Parque Nacional Sajama (Bolivia): Estado poblacional y alternativas de manejo. Mastozoología Neotropical 16(1), 59-67.

[12] Golluscio, R., Deregibus, V., Paruelo, J., 1998. Sustainability and range management in the Patagonian steppes. Ecol. Austral 8, 265-284.

[13] Hanski, I., 1983. Coexistence of competitors in patchy environment. Ecology 64, 1493-500.

[14] Hanski, I., 1994. A practical model of metapopulation dynamics. Journal of Animal Ecology 63(1), 151-162.

[15] Hanski, I., 1998. Metapopulation dynamics. Nature 396, 41-49.

[16] Hanski, I., Schulz, T., Wong, S., Ahola, V., Ruokolainen, A., Ojanen, S., 2017. Ecological and genetic basis of metapopulation persistence of the glanville frutillary butterfly in fragmented landscapes. Nature Communications 8: 14504. URL http://dx.doi.org/10.1038/ncomms14504

[17] Hobbs, N. T., Galvin, K., Stokes, C., Lackett, J., Boone, R., Reid, R., Thornton, P., 2008. Fragmentation of rangelands: implications for humans, animals, and landscapes. Global Environmental Change 18(4), 776-785.

[18] Laguna, M. F., Abramson, G., Kuperman, M. N., Lanata, J. L., Monjeau, J. A., 2015. Mathematical model of livestock and wildlife: Predation and competition under environmental disturbances. Ecological Modelling 309-210, 110-117.

[19] Levins, R., Culver, D., 1971. Regional coexistence of species and competition between rare species. Proc. Natn. Acad. Sci. USA. 68(6), 1246-1248.

[20] Lucca, E. D., 2010. Presencia del puma (Puma concolor) y conflicto con el hombre en las pampas argentinas. Fundación de Historia Natural Felix de Azara, Notas Faunísticas 48, 1-17.

[21] Monjeau, J., 2010. Conservation crossroads and the role of hierarchy in the decision-making process. Natureza \& Conservação 8(2), 112-119.

[22] Monjeau, J., Birney, E., Ghermandi, L., Sikes, R., Margutti, L., Phillips, C., 1998. Plants, small mammals, and the hierarchical landscape classifications in Patagonia. Landscape Ecology 13(5), 285-306.

[23] Musters, G., 2013. At Home with the Patagonians: A Year's Wanderings over Untrodden Ground from the Straits of Magellan to the Rio Negro (Cambridge Library Collection - Anthropology). Cambridge University Press, Cambridge. 
[24] Muñoz, A., 2004. Mammal exploitation in the insular environments of southern South America. In: G.L. Mengoni Goñalons (ed) Zooarchaeology of South America. BAR, International Series 1298. Oxford, UK.

[25] Nabte, M., 2010. Desarrollo de criterios ecológicos para la conservación de mamíferos terrestres en Península Valdés. Ph.D. thesis, Universidad Nacional de Mar del Plata Facultad de Ciencias Exactas y Naturales.

[26] Nabte, M., Marino, A., Rodríguez, M., Monjeau, A., Saba, S., 2013. Range management affects native ungulate populations in Península Valdés, a world natural heritage. PLOS ONE 8(2): e5565. URL https://doi.org/10.1371/journal . pone.0055655

[27] Novaro, A., 1995. Sustainability of harvest of culpeo foxes in Patagonia. Oryx 29, $18-22$.

[28] Ottichilo, W., Grunblatt, J., Said, M., Wargute, P., 2000. Wildlife and livestock population trends in the Kenya rangeland. In: H.H.T. Prins, J.G. Grootenhuis, T.T. Dolan (eds) Wildlife Conservation by Sustainable Use. Conservation Biology Series Vol. 12. Springer, Dordrecht.

[29] Pedrana, J., Bustamante, J., Travaini, A., Rodríguez, A., 2010. Factors influencing guanaco distribution in southern Argentine Patagonia and applications for its sustainable use. Biodiversity \& Conservation 19, 3499-3512.

[30] Prins, H., 2000. Competition between wildlife and livestock in Africa. In: H.H.T. Prins, J.G. Grootenhuis, T.T. Dolan (eds) Wildlife Conservation by Sustainable Use. Conservation Biology Series Vol. 12. Springer, Dordrecht.

[31] Puig, S., Videla, F., Cona, M., Monge, A., 2001. Use of food availability by guanacos (Lama guanicoe) and livestock in northern Patagonia (Mendoza, Argentina). Journal of Arid Environments 47, 291-308.

[32] Savory, A., Butterfield, J., 1988. Holistic management. A new framework for decision making. Island Press.

[33] Solé, R., Bascompte, J., Valls, J., 1992. Stability and complexity of spatially extended two-species competition. J. theor. Biol. 159, 469-480.

[34] Solé, R., Valls, J., 1992. Spiral waves, chaos and multiple attractors in lattice models of interacting populations. Physics Letters A 166, 123-128.

[35] Thungen, J. V., Lanari, M., 2010. Profitability of sheep farming and wildlife management in Patagonia. Pastoralism 1(2), 274-290.

[36] Travaini, A., Zapata, S., Martínez-Peck, R., Delibes, M., 2000. Percepción y actitud humanas hacia la predación de ganado ovino por el zorro colorado (Pseudalopex culpaeus) en Santa Cruz, Patagonia Argentina. Mastozool. Neotrop. 7, $117-129$.

[37] Victurine, R., Curtin, C., 2010. Financial incentives for rangeland conservation. In: J. du Toit, R. Kock, J. Deutsch (eds). Wild rangelands: Conserving Wildlife While Maintaining Livestock in Semi-Arid Ecosystems. Wiley-Blackwell, Oxford.

[38] Wrobel, M., Redford, K., 2010. Introduction: A Review of Rangeland Conservation Issues in an Uncertain Future. In: J.T. du Toit, R. Kock, J. Deutsch (eds). Wild Rangelands: Conserving Wildlife While Maintaining Livestock in Semi-Arid Ecosystems. Wiley-Blackwell, Oxford. 